Supporting Information

\title{
Quantitative analysis of pharmaceutical drugs using a combination of acoustic levitation and high resolution mass spectrometry
}

Sebastian van Wasen, ${ }^{1}$ Yi You ${ }^{2}$, Sebastian Beck, ${ }^{1}$ Jens Riedel, ${ }^{2}$ and Dietrich A. Volmer ${ }^{1 *}$

${ }^{1}$ Humboldt-Universität zu Berlin, Department of Chemistry, Brook-Taylor-Straße 2, 12489 Berlin, Germany

${ }^{2}$ Bundesanstalt für Materialforschung und -prüfung (BAM), Richard-Willstätter-Straße 11, 12489 Berlin, Germany

*Phone: +49 302093 7588. E-mail: Dietrich.Volmer@hu-berlin.de 
Scheme 1. Droplet volume calculation from schlieren imaging

The pixel resolution of the schlieren imaging setup (Figure S1) was determined, yielding a conversion factor of $52.13 \mathrm{pixel} / \mathrm{mm}$; this conversion factor was later used to determine the transient droplet volume in the unit of $\mu \mathrm{L}$. Due to the high refractive index of liquid in contrast to air, the projection of the droplet from the schlieren imaging setup resulted in a dark area of high contrast (Figure S2a). To roughly estimate the droplet projection area, an arbitrary threshold of 120 a.u. was used to generate binary images (Figure S2b).
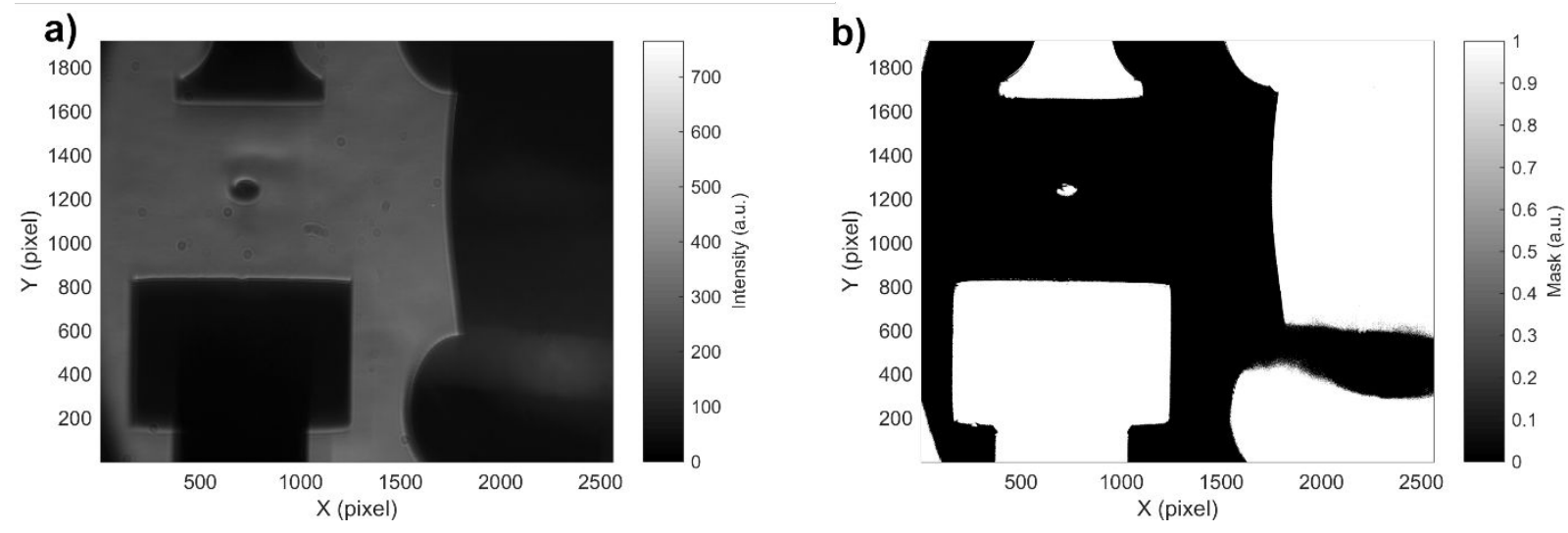

Figure S1 Schlieren image of the levitated droplet (a) and the binary image after thresholding (b).

To estimate the droplet volume from each binary projection, we assumed that the droplet is axially symmetric. Namely, each pixilated slice at a given y is considered a projection of a cylinder with a fixed height of 1 pixel, where the diameter of this cylinder is the number of pixels with a pixel value of 1 . With this approach, the droplet volume can be calculated by:

$$
V=C^{3} \cdot \sum_{Y}\left(2 \pi\left(\left.\frac{1}{2} \sum_{X} I(x, y)\right|_{I=1}\right)\right)
$$

where $V$ is the droplet volume in $\mu \mathrm{L} ; C$ is the conversion factor in pixel $/ \mathrm{mm} ; I(x, y)$ is the binary image. To validate the volume estimation, droplets with known volumes were used to compare with the computed value. For instance, the calculated droplet volume with this computational approach was $7.79 \mu \mathrm{L}$ for an $8.00 \mu \mathrm{L}$ dispensing volume, which suggested a $2.7 \%$ error. 
a)
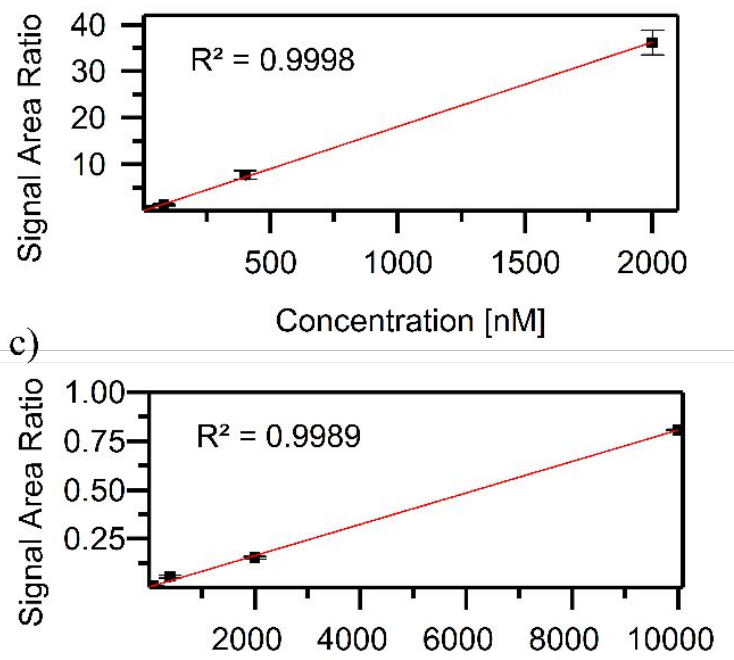

e)

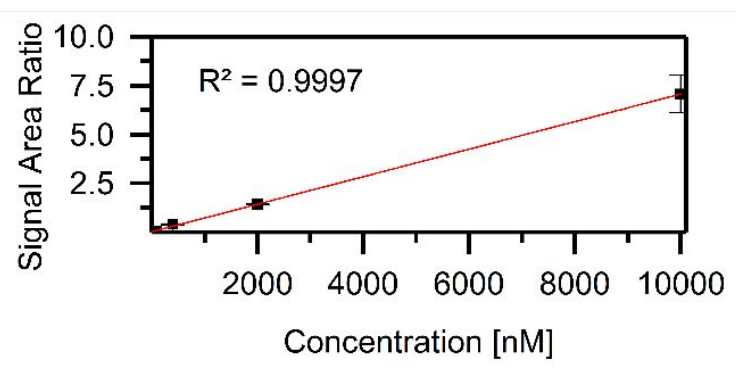

b)

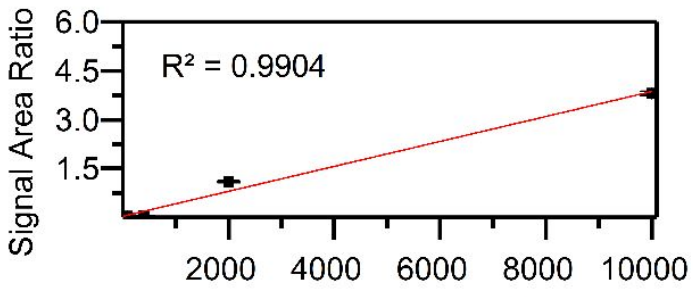

d) Concentration $[\mathrm{nM}]$

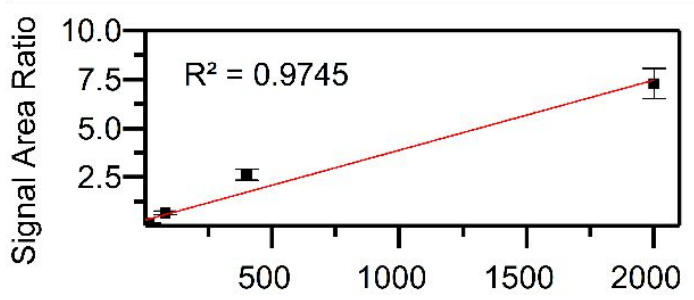

Concentration $[\mathrm{nM}]$

Figure S2 Calibration curves obtained for all investigates pharmaceuticals. A linear range was obtained for a) carbamazepine in the concentration range of $3.2-2,000 \mathrm{nM}$, for b) flumequine in the concentration range of $16.0-10,000 \mathrm{nM}$, for c) trimethoprim in the concentration range of 3.2-10,000 nM, for d) nalidixic acid in the concentration range of 3.2-2,000 $\mathrm{nM}$ and for e) nalidixic acid in the concentration range of 3.2-2,000 nM. Signal area ratio equals area under monoisotopic analyte signal $[\mathrm{M}+\mathrm{H}]^{+}$normalized by the area of the monoisotopic signal of the internal standard (500 nM caffeine). 